\title{
0 steonecrosis maxilar secundaria al uso de bisfosfonatos por vía oral. Exposición de tres casos clínicos relacionados con alendronato
}

\author{
Sacramento Bocanegra-Pérez ${ }^{1}$, Mario Vicente-Barrero르, \\ Manuel Sosa-Henríquez ${ }^{3}$, Arwen Gebaguer Blancoa, \\ Milan Knezevic ${ }^{2}$, José María Castellano-N avarro². \\ O steonecrosis of the jaw secondary to \\ oral alendronate. Report of three cases
}

Osteonecrosis of the jaw associated to biphosphonate use is more common in cancer patients with bone metastases, that are using intravenous diphosphonates. When these drugs are used orally the risk of the complication is lower. We report 3 diabetic women aged 69, 76 and 82 years, receiving alendronate $70 \mathrm{mg}$ every one week. The unveiling event was the extraction of several teeth without the use of antibiotics. All had bone pain, purulent discharge, loss of bone and halitosis. All improved five months after discontinuing alendronate (Rev Méd Chile 2009; 137: 275-9).

(Key words: Alendronates; Biphosphonates; Osteonecrosis)

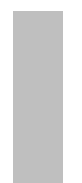

Recibido el 20 de marzo, 2008. Aceptado el 21 de octubre, 2008.

${ }^{1}$ Atención Primaria del Servicio Canario de Salud. ${ }^{2}$ Servicio de Estomatología, Cirugía Oral y Maxilofacial del Hospital Universitario Insular, Las Palmas, España. ${ }^{3}$ Unidad Metabólica Ósea del Hospital Universitario Insular, Las Palmas, España.

${ }^{\text {aOOdontóloga }}$

$\mathrm{L}^{\infty}$ os bisfosfonatos (BF) son análogos de los Lirofosfatos orgánicos, tienen una gran afinidad por los cristales de hidroxiapatita y actúan como potentes inhibidores de la actividad osteoclástica. Los más utilizados son alendronato, risedronato, pamidronato, ácido zoledrónico e ibandronato ${ }^{1}$.

Los BF por vía oral (VO) están recomendados en el tratamiento de la osteoporosis, la osteopenia, la enfermedad de Paget y la osteogénesis

$\overline{\text { Correspondencia a: Dr. Mario Vicente Barrero. c/ Alcalde }}$ Henríquez Pitti 13, 1ำ izq. 35400- Arucas. Las Palmas, España. Teléfono: 928602951 . Fax: 928634736.

E mail: mmvicente@wanadoo.es; mmvicenteb@gmail.com imperfecta en la infancia ${ }^{2,3}$. Aunque la indicación más prevalente es la osteoporosis ${ }^{4,5}$, que puede aparecer en la postmenopausia o en el contexto de otras enfermedades, como la enfermedad inflamatoria intestinal o la cirrosis biliar primaria y por el uso de otros fármacos, principalmente esteroides. No obstante, cualquiera que sea su etiología, los BF VO junto al calcio y la vitamina D juegan un papel fundamental en su tratamiento ${ }^{6}$.

Los BF se toleran bien en general si se administran correctamente, no obstante, se han descrito diferentes efectos adversos y complicaciones asociadas a su uso. Los efectos secundarios más frecuentes de los BF VO son de tipo digestivo: erosiones, ulceraciones crónicas en la mucosa oral $^{7}$, úlceras gástricas, esofagitis y estenosis esofágica ${ }^{8}$. 
La osteonecrosis de los maxilares (ONM) asociada a $\mathrm{BF}$ es una entidad descrita recientemente con numerosas series y casos publicados ${ }^{9-11}$. La mayoría son pacientes oncológicos con metástasis óseas en tratamiento con BF intravenoso, pero también se han publicado un pequeño número de casos relacionados con la terapia de la osteoporosis con $\mathrm{BF} \mathrm{VO}^{1,10-14}$. En ellos, se mencionan que factores locales como enfermedad periodontal, periapical y torus; así como extracciones dentarias recientes, parecen aumentar el riesgo de ONM.

En este trabajo presentamos tres casos de ONM asociados a la administración oral de alendronato.

\section{Casos CLÍNicos}

En la Tabla 1 se encuentran resumidos los tres casos clínicos. La edad de las tres pacientes variaba entre 69 y 82 años; dos habían sido diagnosticadas de osteoporosis y una de artritis reumatoide; no eran fumadoras; las tres pacientes tenían diabetes mellitus no insulina dependiente y coincidían en la ingesta de antidiabéticos orales, calcio, vitamina D y antiagregantes plaquetarios. El caso 1 tenía tratamiento con corticoides y presentó afectación bimaxilar (Figuras 1 y 2). En los tres casos referían buena higiene bucodental, uso esporádico de colutorio antiplaca y en el caso 3 , buen cuidado de sus prótesis parcial superior e inferior; aunque presentaban una importante pérdida de soporte periodontal y escasas piezas dentarias en boca (6, 18 y 12, respectivamente).

La pauta de tratamiento con alendronato fue de 70 miligramos a la semana, excepto en el caso 3 , que previamente, durante 137 meses, se le administró 10 miligramos diarios, para pasar posteriormente a 70 miligramos semanal, durante otros 39 meses.

El factor desencadenante en los tres casos fue la exodoncia de varias piezas dentarias, sin cobertura antibiótica, ni de clorhexidina y sin sutura tras la extracción dental.

La clínica fue similar: hueso expuesto, dolor, supuración, pérdida de hueso, sensación alterada de la mandíbula, halitosis y dificultad para manejo nutricional.

La ortopantomografía mostró principalmente osteolisis en relación con ONM y moderada pérdida de soporte periodontal. La ganmagrafía ósea es muy inespecífica, pues sólo revela captación patológica compatible con ON. El caso 1 fue biopsiado encontrándose intensa inflamación y actinomices. En el caso 3, se realizó tomografía axial computarizada mandibular, encontrándose

Tabla 1. D atos de los pacientes con osteonecrosis maxilar secundarios al tratamiento con biffosfonatos vía oral

\begin{tabular}{|llll|}
\hline & Caso $\mathbf{1}$ & Caso 2 & Caso 3 \\
\hline Sexo & Mujer & Mujer & Mujer \\
Edad & 69 & 82 & 76 \\
Patología base & Artritis reumatoide & Osteoporosis & Osteoporosis \\
Bisfosfonato & Alendronato & Alendronato & Alendronato \\
Vía & Oral & Oral & Oral \\
Dosis & $70 \mathrm{mg}$ & $70 \mathrm{mg}$ & $10 \mathrm{mg} / 70 \mathrm{mg}$ \\
Frecuencia & 7 días & 7 días & diario/7 días \\
Tiempo & $32 \mathrm{meses}$ & 54 meses & 137 meses \\
Dosis total administrada & $8.960 \mathrm{mg}$ & $15.120 \mathrm{mg}$ & 39 meses \\
& & & $41.050 \mathrm{mg}$ \\
Intervención dental & Extracciones & Extracciones & $10.920 \mathrm{mg}$ \\
& $44-45$ y 16 & $35-38$ & Extracciones \\
Localización de ONM & Mandibular y maxilar & Mandibular & Mandibular \\
\hline
\end{tabular}




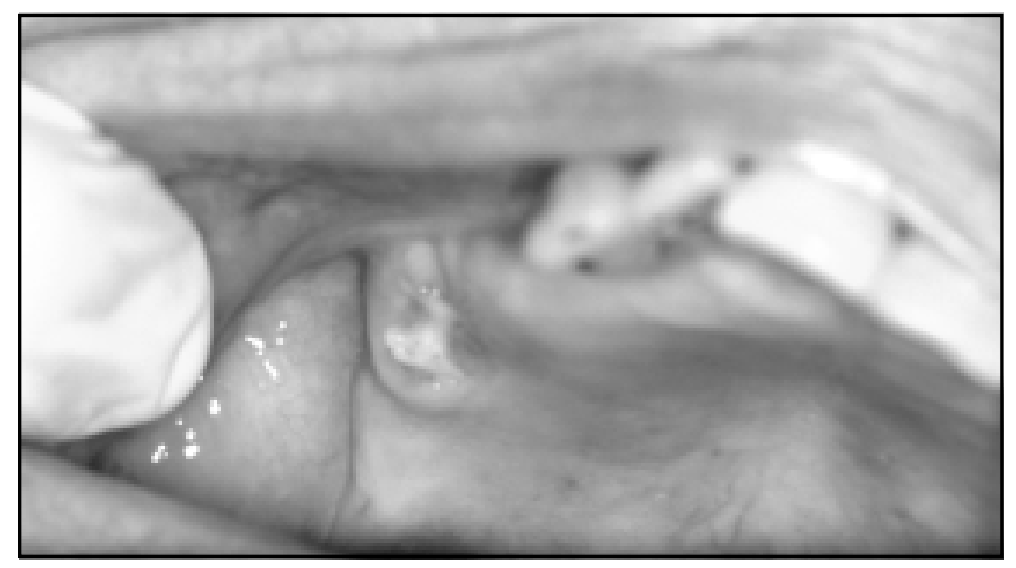

Figura 1. Exposición ósea en maxilar superior.

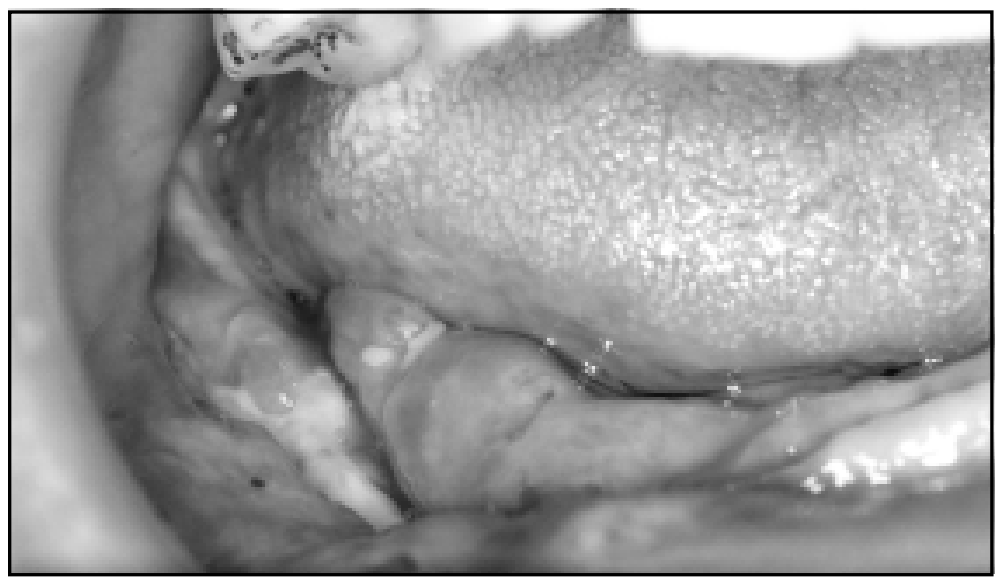

Figura 2. Exposición ósea en maxilar inferior.

lesión lítica de bordes mal delimitados, con áreas de tejido óseo en su interior.

Los tratamientos realizados fueron: curetajes, antibioterapia (amoxi/clavulánico), clorhexidina y ostectomía parcial del proceso alveolar mandibular (caso 1).

La duración promedio con ONM fue de 24 meses (17-36); los tres casos habían mejorado a los 5-6 meses de suspender el BF. Actualmente, no presentan dolor, ni supuración, no toman antibióticos; la exposición ósea es menor, pero no pueden colocar prótesis inferior.

\section{DISCUSIÓN}

Los paneles de expertos reunidos hasta el momento, Asociación Dental Americana, Asociación
Americana de Cirujanos Orales, Sociedad Americana para la Investigación Mineral y Ósea, la Academia Americana de Medicina Oral y el panel español de expertos ${ }^{6,15-18}$ coinciden en que la incidencia exacta y riesgo de ONM son desconocidos y no hay una guía específica para el manejo de los pacientes que toman BF VO.

En la revisión de Woo et al sobre 368 casos de ONM (publicados entre 1966 y 2006), 94\% estaban siendo tratados con BF IV. De ellos, 18 estaban relacionados con alendronato vía oral y en 13 de estos casos se trataba de pacientes con osteoporosis, sin implicaciones oncológicas ${ }^{1}$; pone de manifiesto que $65 \%$ de los casos asienta sólo en la mandíbula, $26 \%$ sólo en el maxilar y 9\% en ambos $^{1}$. Un tercio de estas lesiones eran dolorosas en el momento del diagnóstico y $60 \%$ de los casos 
ocurrían en mujeres. La mayoría de las lesiones se localizaban en la zona posterior de la mandíbula, en la vertiente lingual. Sesenta por ciento de los casos se desencadenó después de una extracción dental o, en general, alguna cirugía dentoalveolar, mientras que el resto de los casos apareció de forma espontánea, a menudo en pacientes portadores de prótesis dental ${ }^{1}$. Estos datos son similares a los encontrados en nuestros casos (Tabla 1).

A finales de 2007, el número total de casos de ONM publicados en pacientes con osteoporosis o enfermedad de Paget era de 64. De ellos, 57 eran para el tratamiento de la osteoporosis y 7 en la enfermedad de Paget. De los 57 casos, sólo 4 eran hombres, aunque el sexo no fue identificado en varios casos y la mayoría fueron tratados con alendronato. La forma de presentación más común fue la exposición del hueso, más infrecuentemente, ulceración de la mucosa, abscesos, fístulas o dolor ${ }^{13,17}$.

Aunque la incidencia de ONM en pacientes que reciben $\mathrm{BF} \mathrm{VO}$ es mucho menor que para $\mathrm{BF}$ $\mathrm{IV}^{9,10,11,15,19}$, el riesgo estimado varía entre $<1 /$ 100.000 y $1 / 10.000$ pacientes-años de tratamiento ${ }^{17}$.

Este riesgo parece aumentar con la duración del tratamiento, especialmente a partir de los tres años. En los casos publicados, la duración mínima del tratamiento era de 2 años. Aunque puede desarrollarse espontáneamente o tras un trauma leve, en general, se relacionan con extracciones dentarias previas; tienen manifestaciones de necrosis menos severas y responden más rápidamente al tratamiento médico específico instaurado (antibioterapia y clorhexidina); suelen presentar mejoría al año tras suspender el $\mathrm{BF}^{14}$, y en nuestra casuística a los 5-6 meses.

Las lesiones pueden necesitar ser tratadas quirúrgicamente, aunque los pacientes deben conocer las posibles complicaciones ${ }^{6}$. Por ello, conforme existan más datos, la estrategia a seguir irá siendo modificada.

En los tres casos que presentamos, dos superaban ampliamente este tiempo de administración con 54 y 176 meses, respectivamente. En el primer caso (32 meses), el uso concomitante de esteroides pudo haber contribuido al desarrollo de ONM, por sus propiedades antiangiogénicas e inmuno- supresoras $^{20}$. Respecto a los antiagregantes plaquetarios, no tenemos otras referencias publicadas donde hayan sido valorados.

En cualquier caso, la ONM se produce por una alteración del metabolismo óseo que llega a comprometer sus propiedades biomecánicas. El trauma, infección o ambos aumentan la demanda para la reparación ósea hasta el punto que excede la capacidad de regeneración, dando lugar a una necrosis ósea localizada. La propiedad antiangiogénica de los BF y otras medicaciones (corticoides), así como la coexistencia de otros factores favorecedores (mal control de diabetes, edad avanzada, fumar, abuso de alcohol), aumentan el riesgo de persistencia y progresión de la necro$\operatorname{sis}^{1,14}$.

\section{Conclusiones}

En aquellos pacientes que hayan tomado de forma continuada BF VO durante más de tres años, con o sin corticoterapia concomitante, habría que valorar suspender la administración de BF tres meses antes y después de la intervención quirúrgi$\mathrm{ca}^{15}$. Pero aunque minimicemos el riesgo (cobertura antibiótica y de clorhexidina, sutura del lecho alveolar, etc.), no podemos eliminar por completo la posibilidad de aparición de ONM.

Es posible que existan muchos más casos que no han sido informados $y$ por lo tanto no confirmados. Por ello, debido al gran número de pacientes recibiendo BF VO para el tratamiento de osteoporosis/osteopenia, es probable que la mayoría de los clínicos puedan encontrar algún paciente con ONM. Es muy importante determinar con exactitud la incidencia de ONM en esta población y conocer el riesgo asociado con el uso a largo plazo (mayor de 3 años) de BF VO. El uso de ciertos coadyuvantes como los corticosteroides, también requieren de un futuro estudio.

En cualquier caso, sería preciso una mayor uniformidad en la publicación de casos, tanto en los criterios diagnósticos como en la información referente a ellos (dosis y duración del tratamiento, localización y duración de las lesiones, hallazgos histopatológicos, etc.). 


\section{REFERENCIAS}

1. Woo SB, Hellstein JW, Kalmar JR. Systematic review: Bisphosphonates and osteonecrosis of the jaws. Ann Intern Med 2006; 144: 753-61.

2. Delmas PD, Meunier PJ. The management of Paget's disease of bone. N Engl J Med 1997; 336: 558-66.

3. Letocha AD, Cintas HL, Troendle JF, Reynolds JC, Cann CE, Chernoff EJ et al. Controlled trial of pamidronate in children with types III and IV osteogenesis imperfecta confirms vertebral gains but not short-term functional improvement. Journal of Bone and Mineral Research 2005; 20: 977-86.

4. Delmas PD. The use of bisphosphonates in the treatment of osteoporosis. Curr Opin Rheumatol 2005; 17: 462-6.

5. WATTS NB. Bisphosphonate treatment of osteoporosis. Clin Geriatr Med 2003; 19: 395-414.

6. AdVISORY TASK FORCE ON BISPHOSPHONATE-RELATED OSTEONECrosis of the jaWs, American Association of Oral. American Association of Oral and Maxillofacial Surgeons position paper on bisphosphonate-related osteonecrosis of the jaws. J Oral Maxillofac Surg 2007 Mar; 6: 369-76.

7. González-Moles MA, Bagán-Sebastián JV. Alendronate-related oral mucosa ulcerations. J Oral Pathol Med 2000; 29: 514-8.

8. Ponte-Fernández N, Estefania-Fresco R, Aguirre-Urizar JM. Bisphosphonates and Oral Pathology I. General and preventive aspects. Med Oral Patol Oral Cir Bucal 2006; 11: E396-400.

9. Migliorati CA, Schubert MM, Peterson De, Seneda lM. Bisphosphonate-associated osteonecrosis of mandibular and maxillary bone: an emerging oral complication of supportive cancer therapy. Cancer 2005; 104: 83-93.

10. Marx RE, Sawatari Y, Fortin M, Broumand V. Bisphosphonateinduced exposed bone (osteonerosis/osteopetrosis) of the jaws: risk factors, recognition, prevention, and treatment. J Oral Maxillofac Surg 2005; 63: 1567-75.

11. Ruggiero SL, Mehrotra B, Rosenberg TJ, Engroff SL. Osteonecrosis of the jaws associated with the use of bisphosphonates: a review of 63 cases. J Oral Maxillofac Surg 2004; 62: 527-34.

12. Brooks JK, Wilson AJ, Sindler AJ, Ashman SG, SchwarTZ KG, Nikitakis NG. Osteonecrosis of the jaws associated with use of risedronate: Report of 2 new cases. Oral Surg Oral Med Oral Pathol Oral Radiol Endod 2007; 103: 780-6.

13. Levin L, Laviv A, Schwartz-Arad D. Denture-related osteonecrosis of the maxilla associated with oral bisphosphonate treatment. J Am Dent Assoc 2007; 138: 1218-20.

14. Malden NJ, Pai AY. Oral bisphosphonate associated osteonecrosis of the jaws: three case reports. $\mathrm{Br}$ Dent J 2007; 203: 93-7.

15. American Dental Association Council On Scientific AffaIRss. Dental management of patients receiving oral bisphosphonate therapy. Expert panel recommendations. J Am Dent Assoc 2006; 137: 1144-50.

16. Bagán JV, Blade J, Cozar JM, Constela M, García Sanz R, Gómez Veiga F et al. Recomendaciones para la prevención, diagnóstico y tratamiento de osteonecrosis de los maxilares (ONM) en pacientes con cáncer tratados con bifosfonatos. Med Oral Patol Oral Cir Bucal 2007; 12: 279-83.

17. Khosla S, Burr D, Cauley J, Dempster DW, Ebeling PR, Felsenberg D et al. Bisphosphonate-Associated Osteonecrosis of the Jaw: Report of a Task. Force of the American Society for Bone and Mineral Research (Editorial). J Bone Miner Res 2007; 22: 1479-91.

18. Migliorati CA, Casiglia J, Epstein J, Jacobsen PL, Siegel MA, Woo SB. Managing the care of patients with bisphosphonate-associated osteonecrosis. An American Academy of Oral Medicine position paper. J Am Dent Assoc 2005; 136: 1658-68.

19. Ruggiero SL, Fantasia J, Carlson E. Bisphosphonaterelated osteonecrosis of the jaw: background and guidelines for diagnosis, staging and management. Oral Surg Oral Med Oral Path Oral Radiol Endod 2006; 102: 433-41.

20. Pires FR, Miranda A, Cardoso ES, Cardosoas, Fregnani ER, Pereira CM et al. Oral avascular bone necrosis associated with chemotherapy and bisphosphonate therapy. Oral Dis 2005; 11: 365-9. 